\title{
TIC Dans L'enseignement Des Mathematiques Au Lycee Marocain
}

\author{
Jamal Harraq \\ Khalid Hattaf
}

Centre Régional des Métiers de l'Education et de la Formation (CRMEF),

Casablanca, Maroc

Laboratoire d'Analyse, Modélisation et Simulation (LAMS),

Faculté des Sciences de Ben M'sik, Université Hassan II, Casablanca, Maroc

\section{Mohamed Bamhamed}

Centre Régional des Métiers de l'Education et de la Formation (CRMEF),

Casablanca, Maroc

Naceur Achtaich

Laboratoire d'Analyse, Modélisation et Simulation (LAMS),

Faculté des Sciences de Ben M'sik, Université Hassan II, Casablanca, Maroc

Doi:10.19044/esj.2018.v14n22p86 URL:http://dx.doi.org/10.19044/esj.2018.v14n22p86

\begin{abstract}
In this work, we investigate the use of information and communication technologies (ICT) in the teaching of mathematics at Moroccan high school. We first start with the analysis of school books from the three years of Baccalaureate. Furthermore, we analyze the pedagogical orientations concerning the use of these technologies. The results obtained from this study show that the use of ICT in mathematics education at Moroccan high school is still too limited.
\end{abstract}

Keywords: Mathematics education, ICT, pedagogical orientations, teaching practices.

\section{Résumé}

Dans ce travail, nous étudions l'utilisation des technologies de l'information et de la communication (TIC) dans l'enseignement des mathématiques au lycée marocain. Nous commençons par l'analyse des manuels scolaires des trois années du Baccalauréat. De plus, nous analysons les orientations pédagogiques concernant l'utilisation de ces technologies. Les résultats obtenus de cette étude montrent que l'utilisation des TIC dans l'enseignement des mathématiques au lycée marocain reste encore très limitée. 
Mots clés: Enseignement des mathématiques, TIC, orientations pédagogiques, pratiques enseignantes.

\section{Introduction:}

Les technologies de l'information et de la communication (TIC) ont plusieurs avantages dans l'enseignement. Elles encouragent la communication et le partage des connaissances. Elles permettent de se concentrer sur des stratégies et des interprétations de résultats plutôt que de passer du temps sur des calculs fastidieux. De plus, les TIC soutiennent la pédagogie constructiviste, dans laquelle les apprenants utilisent la technologie pour explorer et comprendre les concepts mathématiques (Keong et al. 2005). De même, Ittigson et Zewe (2003) ont remarqué que la technologie est essentielle dans l'enseignement et l'apprentissage des mathématiques car elle améliore la compréhension des concepts de base chez les apprenants.

$\mathrm{Vu}$ l'importance des TIC, la charte nationale de l'éducation et de la formation (Royaume du Maroc, 1999) a consacré le levier 10 pour l'usage des nouvelles technologies de l'information et de la communication (NTIC) et elle considère que la technologie pédagogique joue un rôle déterminant et croissant dans les systèmes et méthodes d'enseignement. Pour cela, elle recommande au département de l'État chargé de l'éducation et de la formation d'intégrer ces technologies dans la formation continue et la mise en place de programmes de télé-enseignement et d'équiper les établissements en NTIC. En 2005, le ministère de l'éducation nationale a adopté une stratégie visant la généralisation de l'introduction des TIC au sein de tous les établissements scolaires publics. Cette stratégie s'est traduite par l'adoption du programme GENIE. Ce programme ambitionnait d'équiper, sur une période de trois ans, tous les établissements en salles multimédia connectées à l'Internet.

D'autre part et selon les orientations pédagogiques de 2007 (voir Royaume du Maroc, 2007, page 47), l'outil informatique dans les nouveaux curricula a une considération spéciale, en raison du rôle qu'il occupe dans divers domaines et en tenant compte des orientations et choix énoncés dans la charte nationale de l'éducation et de la formation concernant le champ d'utilisation des NTIC.

L'objectif de ce travail est de présenter une étude sur l'usage des TIC dans l'enseignement des mathématiques au lycée marocain. Pour ce faire, nous analysons les manuels scolaires et les orientations pédagogiques concernant l'utilisation de ces technologies.

\section{Analyse des manuels scolaires:}

D'après la charte nationale de l'éducation et de la formation, le système d'éducation marocain se présente comme suit: Après un préscolaire de deux ans (4-5 ans), les élèves passent 6 ans à l'école primaire (6-11 ans), trois ans 
au collège (12-14 ans) et trois ans au lycée (15-17 ans). Pour avoir un baccalauréat en sciences mathématiques, les élèves doivent passer une année de tronc commun scientifique et technologique (TCST), et deux années de baccalauréat en sciences mathématiques (BSM). Notre étude concerne l'usage des TIC dans l'enseignement des mathématiques durant ces trois années.

Premièrement, nous commençons par l'analyse des manuels de l'année de TCST, reconnus par le ministère de l'éducation nationale (MEN). Il s'agit de Najah fi riyadiyat (MEN, 2006a), Fi rihab riyadiyat (MEN, 2005a) et Wahate riyadiyat (MEN, 2005b). Ces manuels sont notés respectivement $\mathrm{M}_{1}, \mathrm{M}_{2}$ et M3.

L'usage des TIC pour chaque manuel de TCST est donné dans les tableaux 1,2 et 3 . Le nombre d'activités et d'exercices faisant usage des TIC est représenté respectivement dans les figures 1,2 et 3 .

Tableau 1: Usage des TIC dans le manuel $\mathrm{M}_{1}$ du TCST.

\begin{tabular}{|c|c|c|c|c|c|c|c|}
\hline \multirow{2}{*}{\multicolumn{2}{|c|}{ Leçon }} & \multicolumn{3}{|c|}{ Activités } & \multicolumn{3}{|c|}{ Exercices } \\
\hline & & $\begin{array}{l}\text { Sans } \\
\text { TIC }\end{array}$ & $\begin{array}{c}\text { Avec } \\
\text { TIC }\end{array}$ & $\begin{array}{c}\text { Logiciel } \\
\text { utilisé }\end{array}$ & $\begin{array}{c}\text { Sans } \\
\text { TICE }\end{array}$ & $\begin{array}{l}\text { Avec } \\
\text { TICE }\end{array}$ & $\begin{array}{c}\text { Logiciel } \\
\text { utilisé }\end{array}$ \\
\hline 1 & Ensemble $\mathrm{N}$ et Arithmétique & 6 & 0 & - & 64 & $\mathbf{0}$ & - \\
\hline 2 & Ensembles & 5 & 1 & Calculatrice & 85 & 1 & Calculatrice \\
\hline 3 & Ordre dans $\mathbf{R}$ & 6 & 3 & Calculatrice & 133 & 1 & Calculatrice \\
\hline 4 & Polynômes & 7 & $\mathbf{0}$ & - & 84 & $\mathbf{0}$ & - \\
\hline 5 & Equations et inéquations & 16 & $\mathbf{0}$ & - & 114 & $\mathbf{0}$ & - \\
\hline 6 & Calcul vectoriel & 10 & $\mathbf{0}$ & - & 85 & $\mathbf{0}$ & - \\
\hline 7 & Projection & 6 & $\mathbf{0}$ & - & 21 & $\mathbf{0}$ & - \\
\hline 8 & Droite dans le plan & 8 & $\mathbf{0}$ & - & 52 & $\mathbf{0}$ & - \\
\hline 9 & Calcul trigonométrique & 9 & $\mathbf{0}$ & - & 75 & $\mathbf{0}$ & - \\
\hline 10 & Statistique & 4 & 1 & Calculatrice & 26 & $\mathbf{0}$ & - \\
\hline 11 & Transformations usuelles & 7 & $\mathbf{0}$ & - & 47 & $\mathbf{0}$ & - \\
\hline 12 & Produit scalaire & 10 & $\mathbf{0}$ & - & 52 & $\mathbf{0}$ & - \\
\hline 13 & Fonctions & 12 & 3 & $\begin{array}{c}\text { Calculatrice } \\
\text { Excel }\end{array}$ & 86 & $\mathbf{0}$ & - \\
\hline 14 & Géométrie dans l'espace & 11 & $\mathbf{0}$ & - & 63 & $\mathbf{0}$ & - \\
\hline
\end{tabular}



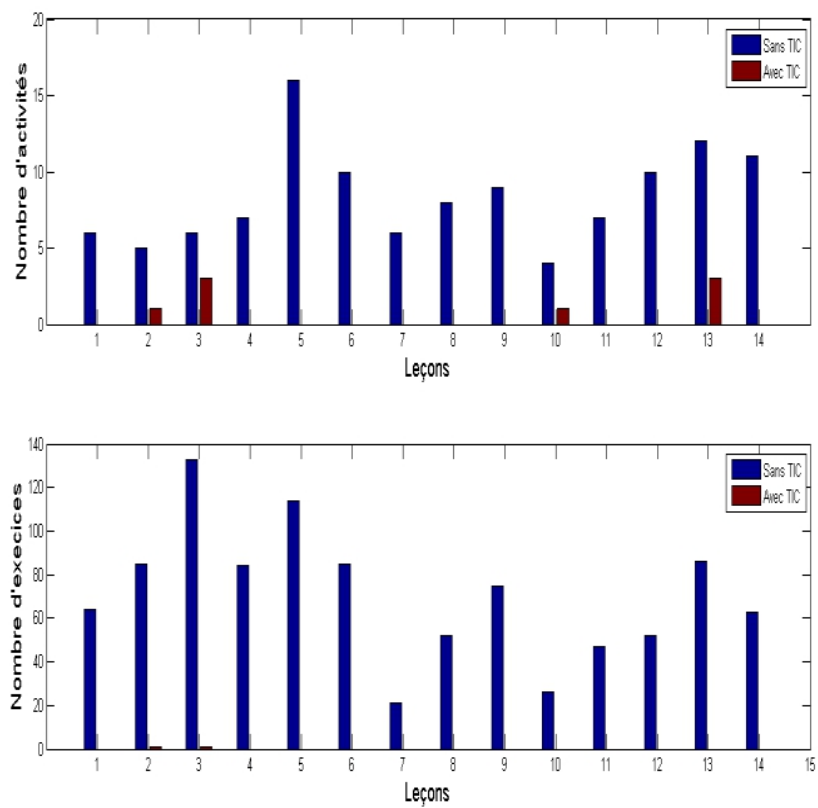

Figure 1: Nombre d'activités et d'exercices avec et sans TIC dans le manuel $\mathrm{M}_{1} \mathrm{du}$ TCST.

Tableau 2 : Usage des TIC dans le manuel $\mathrm{M}_{2}$ du TCST.

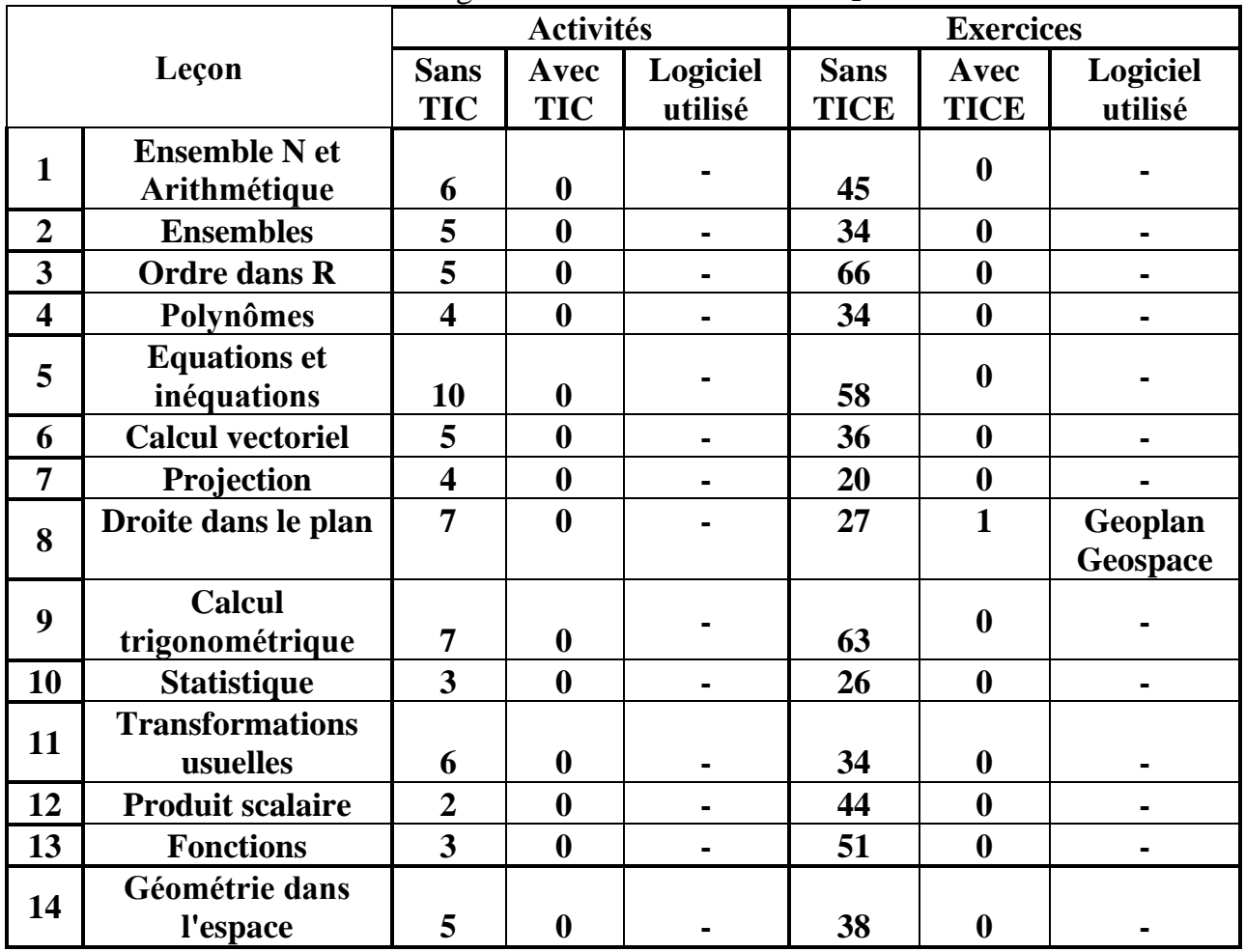



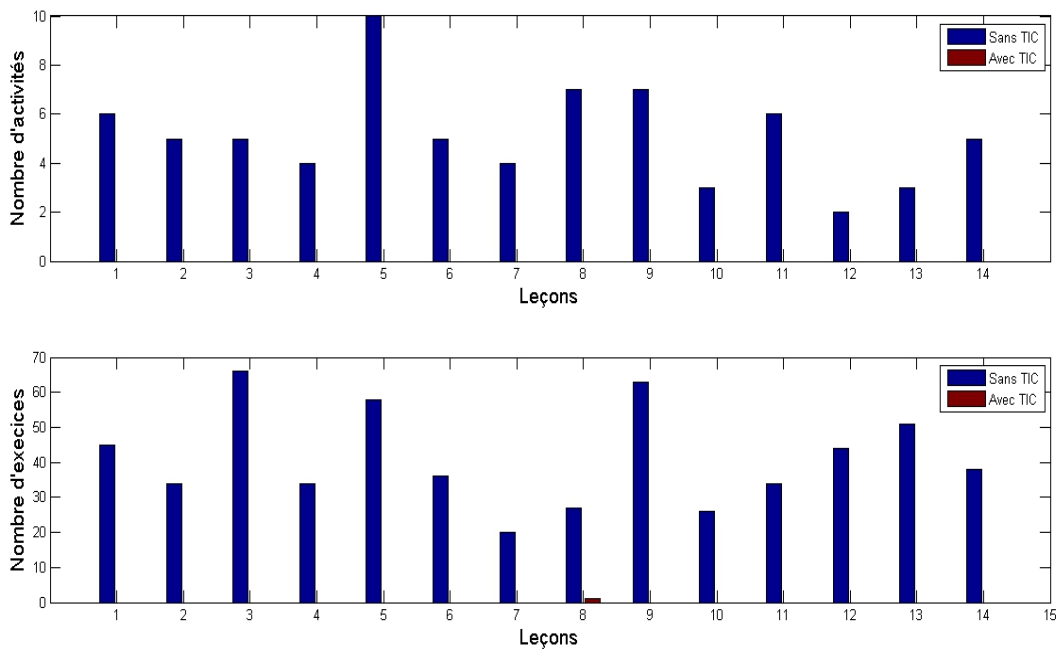

Figure 2: Nombre d'activités et d'exercices avec et sans TIC dans le manuel $\mathrm{M}_{2}$ du TCST.

Tableau 3 : Usage des TIC dans le manuel $\mathrm{M}_{3}$ du TCST.

\begin{tabular}{|c|c|c|c|c|c|c|c|}
\hline \multirow{2}{*}{ Leçon } & \multicolumn{3}{|c|}{ Activités } & \multicolumn{3}{c|}{ Exercices } \\
\cline { 3 - 8 } & $\begin{array}{c}\text { Sans } \\
\text { TIC }\end{array}$ & $\begin{array}{c}\text { Avec } \\
\text { TIC }\end{array}$ & $\begin{array}{c}\text { Logiciel } \\
\text { utilisé }\end{array}$ & $\begin{array}{c}\text { Sans } \\
\text { TICE }\end{array}$ & $\begin{array}{c}\text { Avec } \\
\text { TICE }\end{array}$ & $\begin{array}{c}\text { Logiciel } \\
\text { utilisé }\end{array}$ \\
\hline 1 & $\begin{array}{c}\text { Ensemble N et } \\
\text { Arithmétique }\end{array}$ & 6 & 0 & - & 72 & 0 & - \\
\hline 2 & Ensembles & 6 & 0 & - & 62 & 1 & Calculatrice \\
\hline 3 & Ordre dans R & 8 & 1 & Calculatrice & 72 & 1 & Calculatrice \\
\hline 4 & Polynômes & 3 & 0 & - & 21 & 0 & - \\
\hline 5 & $\begin{array}{c}\text { Equations et } \\
\text { inéquations }\end{array}$ & 8 & 0 & - & 124 & 0 & - \\
\hline 6 & Calcul vectoriel & 5 & 0 & - & 79 & 0 & - \\
\hline 7 & Projection & 8 & 0 & - & 36 & 0 & - \\
\hline 8 & $\begin{array}{c}\text { Droite dans le } \\
\text { plan }\end{array}$ & 7 & 0 & - & 75 & 0 & - \\
\hline 9 & $\begin{array}{c}\text { Calcul } \\
\text { trigonométrique }\end{array}$ & 18 & 0 & - & 93 & 2 & Calculatrice \\
\hline 10 & Statistique & 10 & 0 & - & 38 & 0 & - \\
\hline 11 & $\begin{array}{c}\text { Transformations } \\
\text { usuelles }\end{array}$ & 4 & 0 & - & 28 & 0 & - \\
\hline 12 & Produit scalaire & 4 & 0 & - & 47 & 0 & - \\
\hline 13 & Fonctions & 15 & 0 & - & 113 & 0 & - \\
\hline 14 & $\begin{array}{c}\text { Géométrie dans } \\
\text { l'espace }\end{array}$ & 9 & 0 & - & 70 & 0 & - \\
\hline
\end{tabular}



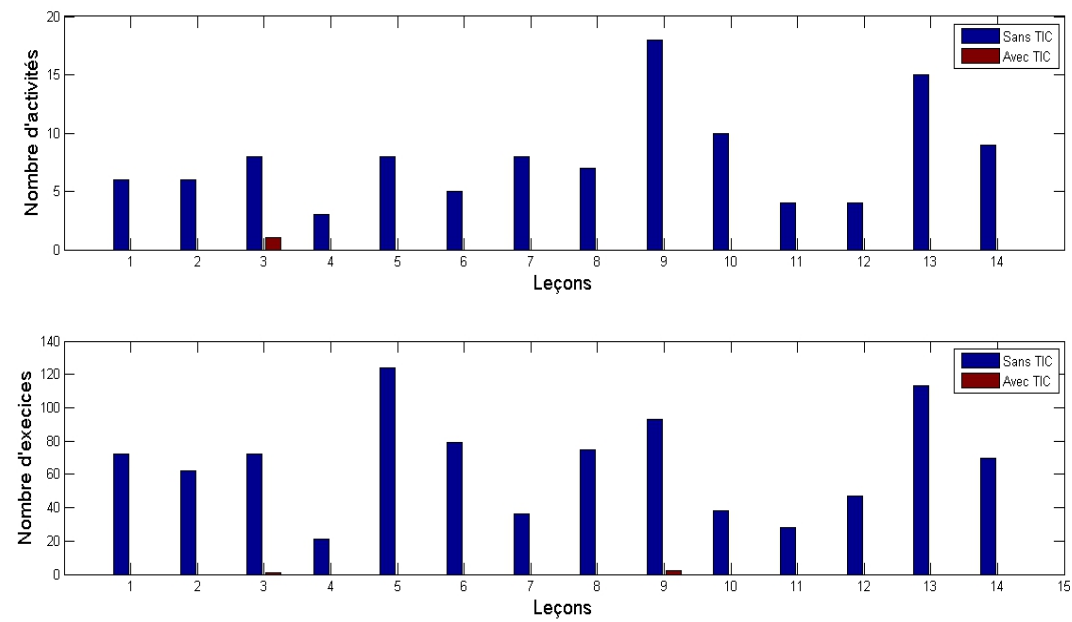

Figure 3: Nombre d'activités et d'exercices avec et sans TIC dans le manuel $\mathrm{M}_{3}$ du TCST.

Par ailleurs, les tableaux et les figures 4,5 et 6 , issus de l'analyse des manuels de la lère année du BSM, à savoir, Mofid fi riyadiyat d'algèbre et géométrie (MEN, 2006b), Wadih fi riyadiyat d'algèbre et géométrie (MEN, 2008a) et Mofid fi riyadiyat d'analyse (MEN, 2007a) montrant le nombre d'activités et d'exercices faisant usage des TIC, constituent la deuxième étape de l'analyse.

Tableau 4 : Usage des TIC dans le manuel $\mathrm{M}_{1}$ du 1BSM.

\begin{tabular}{|c|c|c|c|c|c|c|c|}
\hline \multirow{2}{*}{\multicolumn{2}{|c|}{ Leçon }} & \multicolumn{3}{|c|}{ Activités } & \multicolumn{3}{|c|}{ Exercices } \\
\hline & & & & $\mathbf{I} 0^{\circ}$ & Sans & Avec & \\
\hline 1 & Notions de logioue & 9 & 0 & - & 87 & $\mathbf{0}$ & -2 \\
\hline 2 & Ensembles et applications & 8 & $\mathbf{0}$ & - & 221 & $\mathbf{0}$ & - \\
\hline 3 & Dénombrement & 3 & $\mathbf{0}$ & - & 43 & $\mathbf{0}$ & - \\
\hline 4 & Arithmétique & 7 & $\mathbf{0}$ & - & 125 & $\mathbf{0}$ & - \\
\hline 5 & Barycentre & 7 & $\mathbf{0}$ & - & 86 & 1 & Geoplan \\
\hline 6 & $\begin{array}{c}\text { Produit scalaire dans le } \\
\text { plan }\end{array}$ & 12 & $\mathbf{0}$ & ـ & 110 & 2 & Geoplan \\
\hline 7 & Rotation & 7 & $\mathbf{0}$ & - & 42 & 1 & Geoplan \\
\hline 8 & Vecteurs de l'espace & 5 & $\mathbf{0}$ & - & 66 & 1 & Geospace \\
\hline 9 & Analytique de l'espace & 7 & $\mathbf{0}$ & - & 50 & 1 & Geospace \\
\hline 10 & $\begin{array}{c}\text { Produit scalaire dans } \\
\text { l'espace }\end{array}$ & 4 & $\mathbf{0}$ & - & 45 & $\mathbf{0}$ & - \\
\hline 11 & Produit vectoriel & 4 & $\mathbf{0}$ & - & 41 & $\mathbf{0}$ & - \\
\hline
\end{tabular}



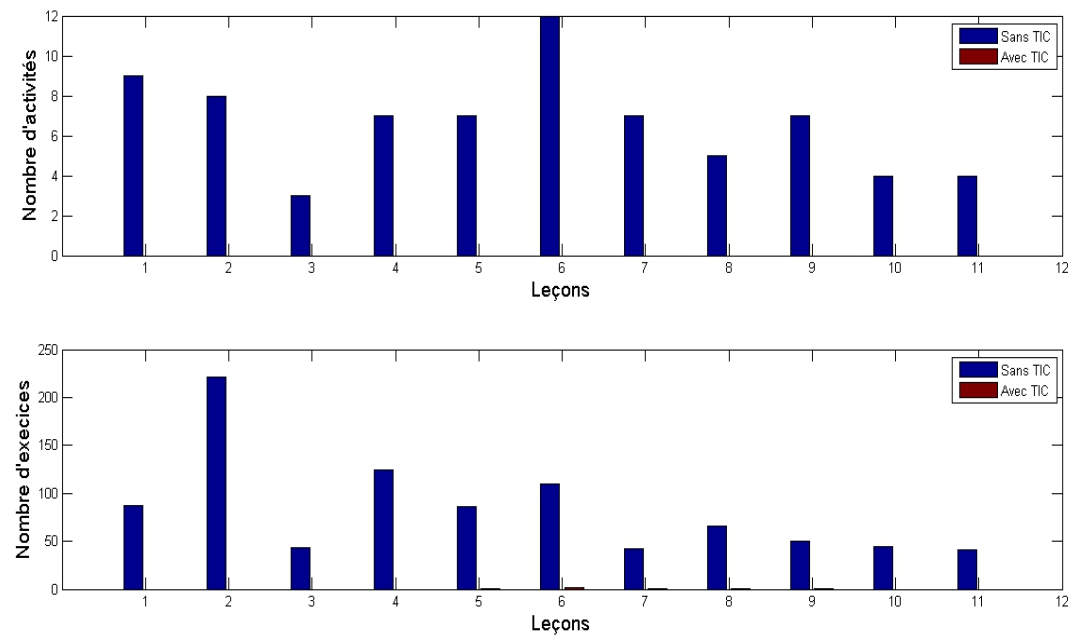

Figure 4: Nombre d'activités et d'exercices avec et sans TIC dans le manuel $\mathrm{M}_{1}$ du 1BSM.

Tableau 5 : Usage des TIC dans le manuel $\mathrm{M}_{2}$ du 1BSM.

\begin{tabular}{|c|c|c|c|c|c|c|c|}
\hline \multirow{2}{*}{\multicolumn{2}{|c|}{ Leçon }} & \multicolumn{3}{|c|}{ Activités } & \multicolumn{3}{|c|}{ Exercices } \\
\hline & & \multirow{3}{*}{$\begin{array}{c}\text { Sans } \\
\text { TIC } \\
15\end{array}$} & \multirow{3}{*}{$\begin{array}{c}\text { Ave } \\
\text { c } \\
\text { TIC } \\
0\end{array}$} & \multirow{3}{*}{$\begin{array}{c}\begin{array}{c}\text { Logiciel } \\
\text { utilisé }\end{array} \\
-\end{array}$} & \multirow{3}{*}{$\begin{array}{c}\text { Sans } \\
\text { TIC } \\
\mathbf{E} \\
60\end{array}$} & \multirow{3}{*}{$\begin{array}{c}\text { Ave } \\
\text { c } \\
\text { TIC } \\
\text { E } \\
0\end{array}$} & \multirow{3}{*}{$\begin{array}{c}\begin{array}{c}\text { Logiciel } \\
\text { utilisé }\end{array} \\
-\end{array}$} \\
\hline & & & & & & & \\
\hline 1 & Notions de logique & & & & & & \\
\hline 2 & $\begin{array}{c}\text { Ensembles et } \\
\text { applications }\end{array}$ & 8 & $\mathbf{0}$ & - & 66 & $\mathbf{0}$ & - \\
\hline 3 & Dénombrement & 7 & $\mathbf{0}$ & - & 43 & $\mathbf{0}$ & - \\
\hline 4 & Arithmétique & 7 & $\mathbf{0}$ & - & 78 & 2 & $\begin{array}{c}\text { Excel } \\
\text { Calculatri } \\
\text { ce }\end{array}$ \\
\hline 5 & Barycentre & 5 & $\mathbf{0}$ & - & 45 & 1 & Geoplan \\
\hline 6 & $\begin{array}{c}\text { Produit scalaire dans le } \\
\text { plan }\end{array}$ & 11 & $\mathbf{0}$ & - & 54 & 1 & Geoplan \\
\hline 7 & Rotation & 7 & $\mathbf{0}$ & - & 44 & 1 & Geoplan \\
\hline 8 & Vecteur de l'espace & 4 & $\mathbf{0}$ & - & 36 & 1 & Geospace \\
\hline 9 & Analytique de l'espace & 8 & $\mathbf{0}$ & - & 44 & 1 & Geospace \\
\hline 10 & $\begin{array}{c}\text { Produit scalaire dans } \\
\text { l'espace }\end{array}$ & 3 & $\mathbf{0}$ & - & 47 & $\mathbf{0}$ & \\
\hline 11 & Produit vectoriel & 5 & $\mathbf{0}$ & - & 37 & $\mathbf{0}$ & \\
\hline
\end{tabular}



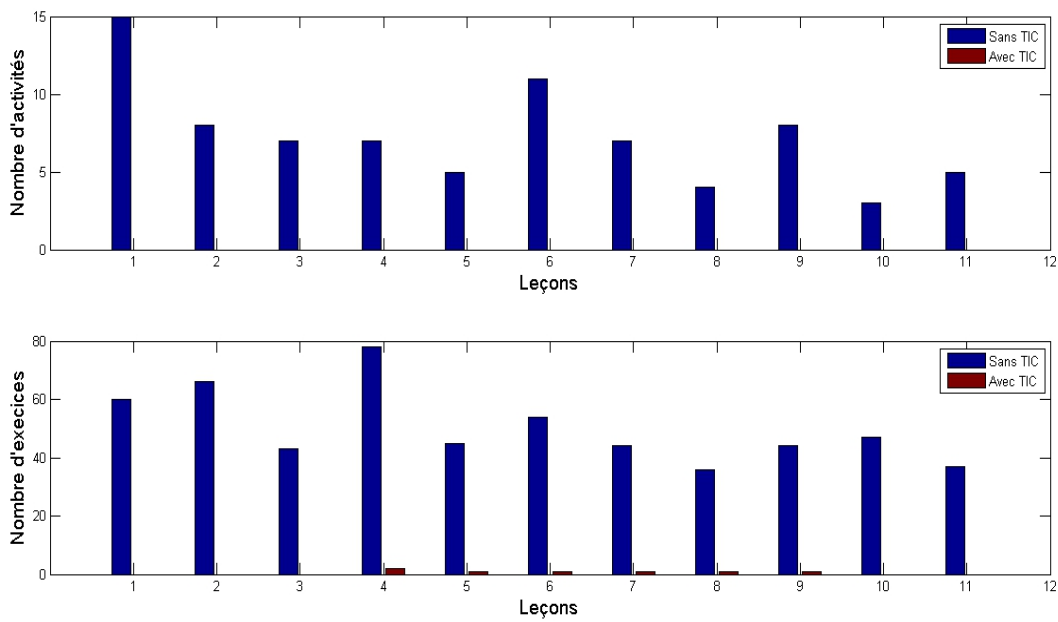

Figure 5: Nombre d'activités et d'exercices avec et sans TIC dans le manuel $\mathrm{M}_{2}$ du 1BSM.

Tableau 6 : Usage des TIC dans le manuel $\mathrm{M}_{3}$ du 1BSM.

\begin{tabular}{|c|c|c|c|c|c|c|c|}
\hline \multirow{2}{*}{ Leçon } & \multicolumn{3}{|c|}{ Activités } & \multicolumn{3}{c|}{ Exercices } \\
\cline { 2 - 8 } & $\begin{array}{c}\text { Sans } \\
\text { TIC }\end{array}$ & $\begin{array}{c}\text { Avec } \\
\text { TIC }\end{array}$ & $\begin{array}{c}\text { Logiciel } \\
\text { utilisé }\end{array}$ & $\begin{array}{c}\text { Sans } \\
\text { TICE }\end{array}$ & $\begin{array}{c}\text { Avec } \\
\text { TICE }\end{array}$ & $\begin{array}{c}\text { Logiciel } \\
\text { utilisé }\end{array}$ \\
\hline 1 & $\begin{array}{c}\text { Généralités sur les } \\
\text { fonctions }\end{array}$ & 8 & 0 & - & 114 & 2 & $\begin{array}{c}\text { Excel } \\
\text { Calculatrice }\end{array}$ \\
\hline 2 & Suites numériques & 4 & 0 & - & 57 & 2 & $\begin{array}{c}\text { Excel } \\
\text { Calculatrice }\end{array}$ \\
\hline 3 & $\begin{array}{c}\text { Calcul } \\
\text { trigonométrique }\end{array}$ & 3 & 0 & - & 121 & 1 & $\begin{array}{c}\text { Cabri- } \\
\text { Geomètre }\end{array}$ \\
\hline 4 & $\begin{array}{c}\text { Limites d'une } \\
\text { fonction numérique }\end{array}$ & 9 & 0 & - & 60 & 2 & $\begin{array}{c}\text { Calculatrice } \\
\text { Geoplan }\end{array}$ \\
\hline 5 & $\begin{array}{c}\text { Dérivabilité } \\
5\end{array}$ & 8 & 0 & - & 104 & 2 & $\begin{array}{c}\text { Sinéquanon } \\
\text { Calculatrice }\end{array}$ \\
\hline 6 & Etude de fonctions & 4 & 0 & - & 57 & 1 & $\begin{array}{c}\text { Archimède } \\
\text { II }\end{array}$ \\
\hline
\end{tabular}



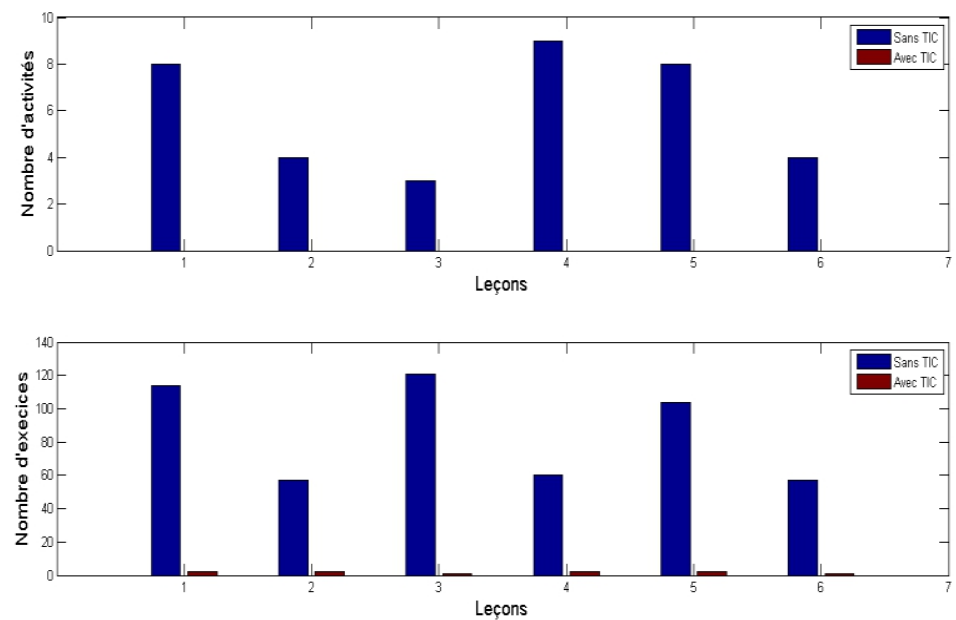

Figure 6: Nombre d'activités et d'exercices avec et sans TIC dans le manuel $\mathrm{M}_{3} \mathrm{du}$ 1BSM.

Finalement, nous analysons les manuels de la 2ème année du BSM. Ces manuels sont Mofid fi riyadiyat d'algèbre et géométrie (MEN, 2009), Wadih fi riyadiyat d'algèbre et géométrie (MEN, 2008b), Mofid fi riyadiyat d'analyse (MEN, 2007b), et Mostakbal fi riyadiyat d'analyse (MEN, 2008c).

Tableau 7 : Usage des TIC dans le manuel $\mathrm{M}_{1}$ du 2BSM.

\begin{tabular}{|c|c|c|c|c|c|c|c|}
\hline \multirow{2}{*}{ Leçon } & \multicolumn{3}{c|}{ Activités } & \multicolumn{3}{c|}{ Exercices } \\
\cline { 2 - 8 } & $\begin{array}{c}\text { Sans } \\
\text { TIC }\end{array}$ & $\begin{array}{c}\text { Avec } \\
\text { TIC }\end{array}$ & $\begin{array}{c}\text { Logiciel } \\
\text { utilisé }\end{array}$ & $\begin{array}{c}\text { Sans } \\
\text { TICE }\end{array}$ & $\begin{array}{c}\text { Avec } \\
\text { TICE }\end{array}$ & $\begin{array}{c}\text { Logiciel } \\
\text { utilisé }\end{array}$ \\
\hline 1 & Nombres complexes & 17 & 0 & - & 101 & 0 & - \\
\hline 2 & Arithmétique & 12 & 0 & - & 151 & 1 & Excel \\
\hline 3 & $\begin{array}{c}\text { Calcul des } \\
\text { probabilités }\end{array}$ & 10 & 1 & Excel & 65 & 1 & Excel \\
\hline 4 & $\begin{array}{c}\text { Loi de composition } \\
\text { interne }\end{array}$ & 8 & 0 & - & 60 & 1 & Excel \\
\hline 5 & $\begin{array}{c}\text { Groupe-Anneaux- } \\
\text { Corps }\end{array}$ & 9 & 0 & - & 55 & 0 & - \\
\hline 6 & $\begin{array}{c}\text { Espace vectoriel } \\
\text { réel }\end{array}$ & 10 & 0 & - & 58 & 0 & - \\
\hline
\end{tabular}



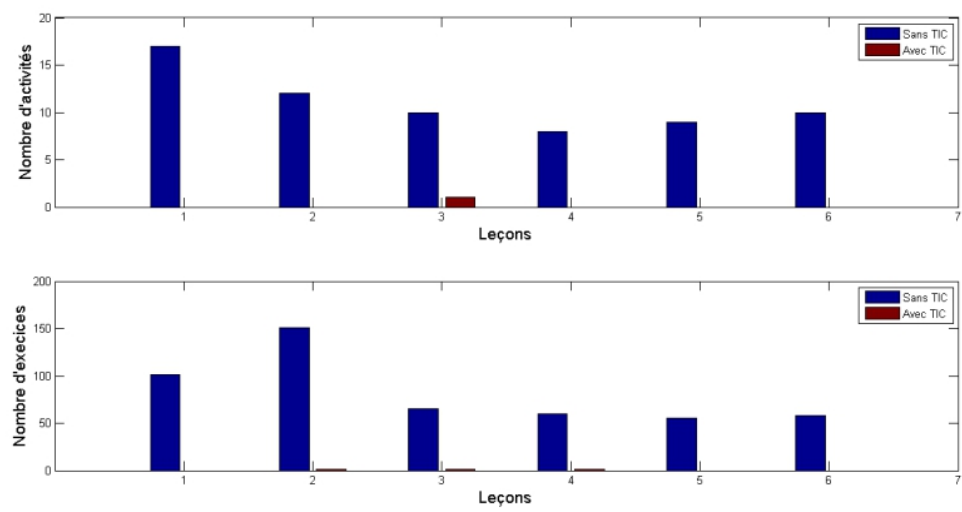

Figure 7: Nombre d'activités et d'exercices avec et sans TIC dans le manuel $\mathrm{M}_{1}$ du 2BSM.

Tableau 8 : Usage des TIC dans le manuel $\mathrm{M}_{2}$ du 2BSM.

\begin{tabular}{|c|c|c|c|c|c|c|c|}
\hline \multirow{2}{*}{ Leçon } & \multicolumn{3}{|c|}{ Activités } & \multicolumn{3}{c|}{ Exercices } \\
\cline { 2 - 8 } & $\begin{array}{c}\text { Sans } \\
\text { TIC }\end{array}$ & $\begin{array}{c}\text { Avec } \\
\text { TIC }\end{array}$ & $\begin{array}{c}\text { Logiciel } \\
\text { utilisé }\end{array}$ & $\begin{array}{c}\text { Sans } \\
\text { TICE }\end{array}$ & $\begin{array}{c}\text { Avec } \\
\text { TICE }\end{array}$ & $\begin{array}{c}\text { Logiciel } \\
\text { utilisé }\end{array}$ \\
\hline 1 & $\begin{array}{c}\text { Nombres } \\
\text { complexes }\end{array}$ & 12 & 0 & - & 86 & 1 & Calculatrice \\
\hline 2 & Arithmétique & 6 & 0 & - & 103 & 1 & Excel \\
\hline 3 & $\begin{array}{c}\text { Calcul des } \\
\text { probabilités }\end{array}$ & 3 & 1 & $\begin{array}{c}\text { Calculatrice } \\
\text { Excel }\end{array}$ & 53 & 0 & - \\
\hline 4 & $\begin{array}{c}\text { Loi de } \\
\text { composition } \\
\text { interne Groupe }\end{array}$ & 5 & 0 & - & 47 & 1 & $\begin{array}{c}\text { Excel } \\
\text { Calculatrice }\end{array}$ \\
\hline 5 & $\begin{array}{c}\text { Anneau - Corps - } \\
\text { Espace vectoriel } \\
\text { réel }\end{array}$ & 5 & 0 & - & 45 & 0 & - \\
\hline
\end{tabular}
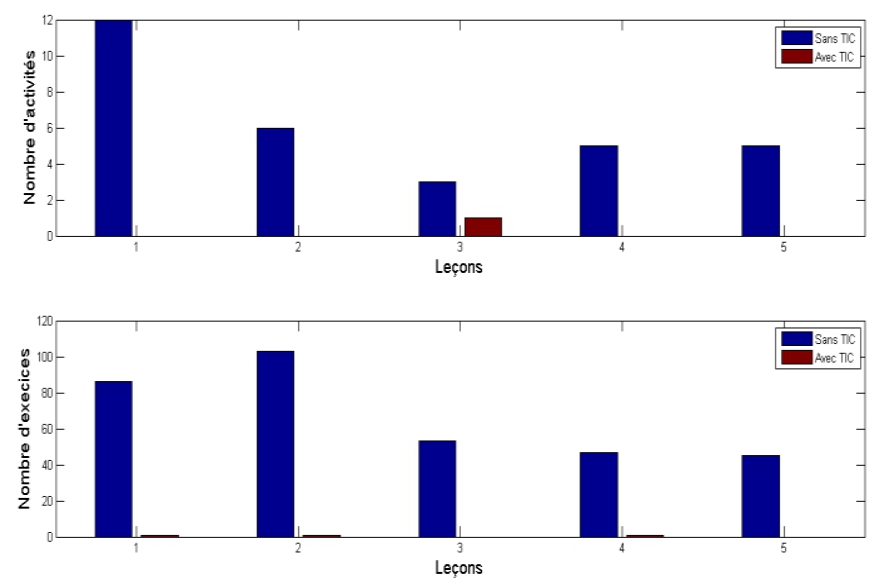

Figure 8: Nombre d'activités et d'exercices avec et sans TIC dans le manuel $\mathrm{M}_{2}$ du 2BSM. 
Tableau 9 : Usage des TIC dans le manuel $\mathrm{M}_{3}$ du 2BSM.

\begin{tabular}{|c|c|c|c|c|c|c|c|}
\hline \multirow{2}{*}{\multicolumn{2}{|c|}{ Leçon }} & \multicolumn{3}{|c|}{ Activités } & \multicolumn{3}{c|}{ Exercices } \\
\cline { 3 - 8 } & $\begin{array}{c}\text { Sans } \\
\text { TIC }\end{array}$ & $\begin{array}{c}\text { Avec } \\
\text { TIC }\end{array}$ & $\begin{array}{c}\text { Logiciel } \\
\text { utilisé }\end{array}$ & $\begin{array}{c}\text { Sans } \\
\text { TICE }\end{array}$ & $\begin{array}{c}\text { Avec } \\
\text { TICE }\end{array}$ & $\begin{array}{c}\text { Logiciel } \\
\text { utilisé }\end{array}$ \\
\hline 1 & Limite et continuité & 10 & 1 & Calculatrice & 98 & 1 & Excel \\
\hline 2 & Suites numeriques & 14 & 0 & - & 65 & 1 & Excel \\
\hline 3 & $\begin{array}{c}\text { Dérivabilité-Etude } \\
\text { des fonctions- } \\
\text { Primitives }\end{array}$ & 11 & 0 & - & 107 & 1 & Excel \\
\hline 4 & $\begin{array}{c}\text { Fonctions } \\
\text { logarithmes }\end{array}$ & 2 & 1 & Calculatrice & 76 & 1 & Excel \\
\hline 5 & $\begin{array}{c}\text { Fonctions } \\
\text { exponentiels }\end{array}$ & 5 & 0 & - & 66 & 1 & Excel \\
\hline 6 & $\begin{array}{c}\text { Théroème des } \\
\text { acroissements finis }\end{array}$ & 4 & 0 & - & 80 & 1 & Excel \\
\hline 7 & $\begin{array}{c}\text { Equations } \\
\text { différentielles }\end{array}$ & 7 & 0 & - & 23 & 1 & Excel \\
\hline 8 & Calcul intégral & 12 & 0 & - & 90 & 1 & Excel \\
\hline
\end{tabular}
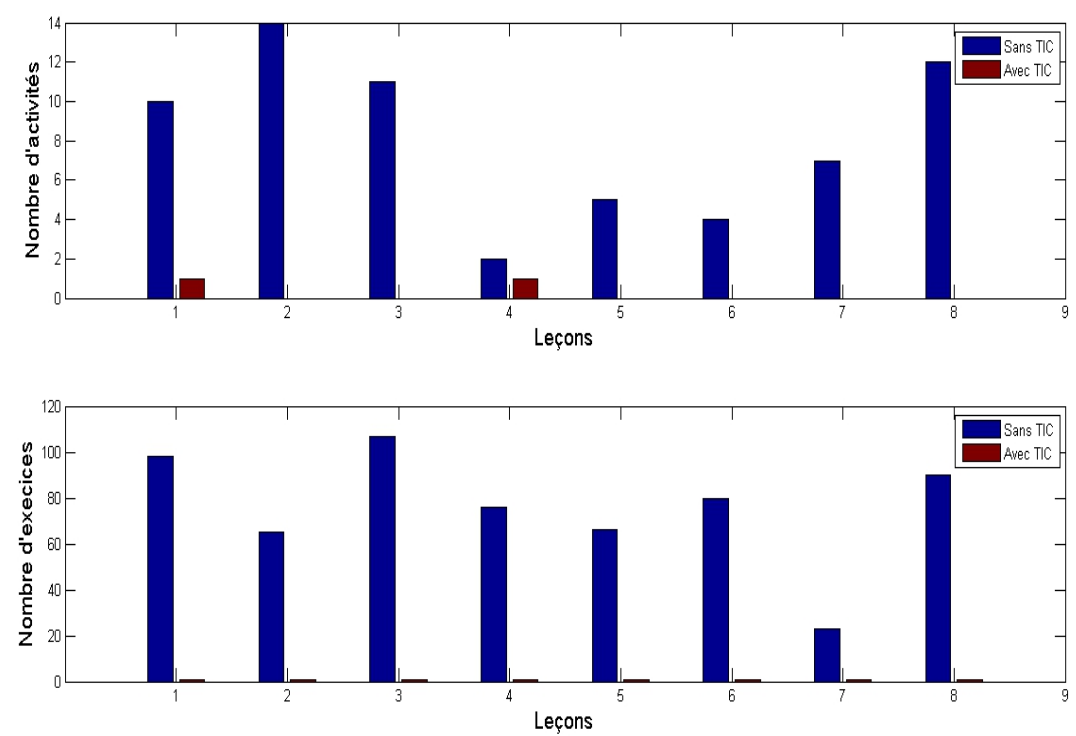

Figure 9: Nombre d'activités et d'exercices avec et sans TIC dans le manuel $\mathrm{M}_{3}$ du 2BSM. 
Tableau 10 : Usage des TIC dans le manuel $\mathrm{M}_{4}$ du 2BSM.

\begin{tabular}{|c|c|c|c|c|c|c|c|}
\hline \multirow{2}{*}{ Leçon } & \multicolumn{3}{|c|}{ Activités } & \multicolumn{3}{c|}{ Exercices } \\
\cline { 2 - 8 } & $\begin{array}{c}\text { Sans } \\
\text { TIC }\end{array}$ & $\begin{array}{c}\text { Avec } \\
\text { TIC }\end{array}$ & $\begin{array}{c}\text { Logiciel } \\
\text { utilisé }\end{array}$ & $\begin{array}{c}\text { Sans } \\
\text { TICE }\end{array}$ & $\begin{array}{c}\text { Avec } \\
\text { TICE }\end{array}$ & $\begin{array}{c}\text { Logiciel } \\
\text { utilisé }\end{array}$ \\
\hline 1 & $\begin{array}{c}\text { Limite et } \\
\text { continuité }\end{array}$ & 6 & 0 & - & 74 & 1 & Excel \\
\hline 2 & $\begin{array}{c}\text { Dérivabilité et } \\
\text { applications }\end{array}$ & 4 & 0 & - & 58 & 0 & - \\
\hline 3 & Limites d'une suite & 6 & 0 & - & 45 & 0 & - \\
\hline 4 & $\begin{array}{c}\text { Etude des } \\
\text { fonctions } \\
\text { numériques }\end{array}$ & 4 & 0 & - & 32 & 0 & - \\
\hline 5 & $\begin{array}{c}\text { Fonctions } \\
\text { primitives }\end{array}$ & 2 & 0 & - & 21 & 0 & - \\
\hline 6 & $\begin{array}{c}\text { Fonctions } \\
\text { logarithmes }\end{array}$ & 5 & 0 & - & 44 & 0 & - \\
\hline 7 & $\begin{array}{c}\text { Fonctions } \\
\text { exponentiels }\end{array}$ & 4 & 0 & - & 48 & 0 & - \\
\hline 8 & Calcul intégral & 6 & 0 & - & 63 & 0 & - \\
\hline 9 & $\begin{array}{c}\text { Equations } \\
\text { différentielles }\end{array}$ & 5 & 0 & - & 30 & 0 & - \\
\hline
\end{tabular}
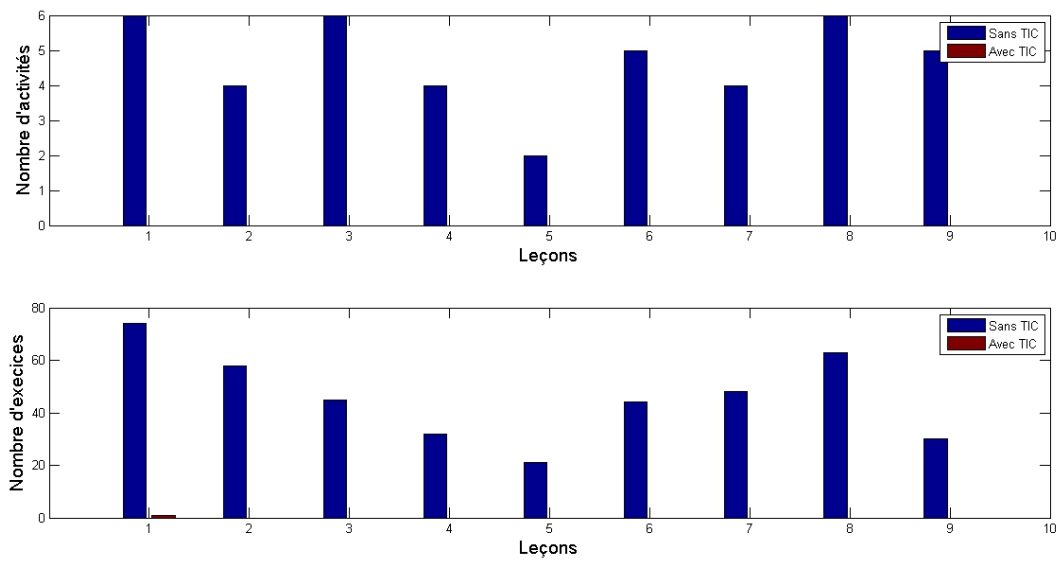

Figure 10: Nombre d'activités et d'exercices avec et sans TIC dans le manuel $\mathrm{M}_{4}$ du 2BSM.

\section{Analyse des orientations pédagogiques:}

Selon les orientations pédagogiques de 2007, les NTIC sont considérées, en plus du tableau et des manuels, parmi les outils pédagogiques. Parmi les spécifications liées aux compétences et contenus, que l'élève doit avoir à la fin du cycle secondaire qualifiant, figure sa capacité d'utiliser les NTIC dans différents domaines de ses études, et d'échanger les informations. De plus, les outils informatiques tels que la calculatrice usuelle, scientifique, 
programmable ou l'ordinateur, grâce aux logiciels qui y sont incorporés, permettent de :

$>$ Simplifier certains types de calcul et déterminer des valeurs approchées;

Vérifier certains résultats;

$>$ Poser et s'assurer des conjectures;

$>$ Traiter des tâches et résoudre des problèmes demandant beaucoup de temps manuellement;

$>$ Construire des tableaux, diagrammes, courbes et des formes géométriques dans le plan et l'espace, ainsi que leurs sections;

$>$ Effectuer des simulations et faire bouger des formes du plan et de l'espace; etc (voir Royaume du Maroc, 2007, page 10).

Dans ce qui suit, nous exposons les orientations pédagogiques concernant l'utilisation des TIC pour chaque année et chaque leçon.

\begin{tabular}{|c|c|c|}
\hline Leçon de TCST & Orientations pédagogiques & Pages \\
\hline Ordre dans IR & $\begin{array}{c}\text { La calculatrice est un bon outil pour l'enseignement } \\
\text { des concepts et notions (encadrement, approximation, } \\
\text { …). Il faut s'assurer que les élèves maîtrisent } \\
\text { l'eriture scientifique d'un nombre et sont conscients } \\
\text { du fait que la calculatrice donne souvent une } \\
\text { approximation décimale du résultat; c'est pourquoi, il } \\
\text { est nécessaire de leur faire acquérir certaines } \\
\text { techniques de la calculatrice scientifique (priorité des } \\
\text { opérations, fonctions des touches, ..). }\end{array}$ & 19 \\
\hline Géométrie dans l'espace & $\begin{array}{c}\text { On peut utiliser les logiciels intégrés à l'ordinateur, } \\
\text { dans la limite de l'existant dans les établissements } \\
\text { scolaires, pour déterminer les sections planes de } \\
\text { certains solides dans l'espace. }\end{array}$ & 24 \\
\hline Fonctions numériques & $\begin{array}{c}\text { On peut utiliser, la calculatrice scientifique pour } \\
\text { déterminer les images, et la calculatrice } \\
\text { programmable pour le traçage des courbes. }\end{array}$ & 25 \\
\hline
\end{tabular}

Remarque: L'utilisation de la calculatrice scientifique pour déterminer la valeur approchée d'un angle dont on connaît le sinus, le cosinus ou la tangente, et inversement ( i.e., déterminer des valeurs approchées du sinus, du cosinus et de la tangente d'un angle donné), a été considérée comme capacité attendue dans la leçon de calcul trigonométrique du TCST (voir, Royaume du Maroc, 2007, page 26).

\begin{tabular}{|c|c|c|}
\hline Leçon de 1BSM & Orientations pédagogiques & Pages \\
\hline $\begin{array}{c}\text { Généralités sur les } \\
\text { fonctions }\end{array}$ & $\begin{array}{c}\text { On peut utiliser les calculatrices et les logiciels } \\
\text { intégrés à l'ordinateur dans l'étude des fonctions. }\end{array}$ & 59 \\
\hline $\begin{array}{c}\text { Généralités sur les } \\
\text { suites numériques }\end{array}$ & $\begin{array}{c}\text { La leçon de suites numériques constitue une } \\
\text { occasion pour habituer les élèves à l'usage des } \\
\text { outils informatiques. }\end{array}$ & 60 \\
\hline
\end{tabular}




\begin{tabular}{|c|c|c|}
\hline Leçon de 2BSM & Orientations pédagogiques & Pages \\
\hline Suites numériques & $\begin{array}{c}\text { Approximation de la notion de limite d'une } \\
\text { suite par l'utilisation du programme Excel. }\end{array}$ & 107 \\
\hline $\begin{array}{c}\text { Calcul des } \\
\text { probabilités }\end{array}$ & $\begin{array}{c}\text { La probabilité d'un événement est introduite } \\
\text { à partir de la stabilité de sa fréquence, et ce } \\
\text { en utilisant la touche rand de la calculatrice } \\
\text { scientifique, ou celle programmable, ou les } \\
\text { logiciels intégrés à l'ordinateur. }\end{array}$ & 116 \\
\hline
\end{tabular}

\section{Conclusion:}

Dans cette étude, nous avons analysé 10 manuels scolaires au lycée concernant l'utilisation des TIC. Il est à noter que les propositions d'usage de ces technologies sont extrêmement limitées et ne dépassent guère quelques cas d'intégration de la calculatrice ou du tableur Excel. Cette remarque est valable aussi pour les orientations pédagogiques qui ne dépassent aucunement la recommandation de l'usage de ces deux outils.

Les pratiques enseignantes désignent tout ce que l'enseignant met en œuvre avant, pendant, voire après la classe (conceptions activées au moment de la préparation des séances, connaissances diverses, discours mathématique et non mathématique pendant la classe, gestes spécifiques, etc) (Robert, 1999). L'analyse de ces pratiques concernant l'utilisation des TIC dans l'enseignement des mathématiques fera l'objet de nos travaux de recherche futurs.

\section{References:}

1. Keong, C. C., Horani, S., \& Daniel, J. (2005). A study on the use of ICT in mathematics teaching. Malaysian Online Journal of Instructional Technology, 2(3), 43-51.

2. Ittigson, R. J., \& Zewe, J. G. (2003). Technology in the mathematics classroom. In Challenges of teaching with technology across the curriculum: Issues and solutions (pp. 114-133). IGI Global.

3. Royaume du Maroc (1999). Charte nationale d'éducation et de formation. Commission Spécial Education-Formation.

4. Royaume du Maroc (2007). Orientations pédagogiques et curricula pour l'enseignement des mathématiques dans le cycle secondaire qualifiant. Ministère de l'éducation nationale, de l'enseignement supérieur et de la recherche scientifique.

5. MEN (2006a). Najah fi riyadiyat, Tronc commun scientifique et technologique. Najah Al Jadida.

6. MEN (2005a). Fi rihab riyadiyat, Tronc commun scientifique et technologique. Essalam Al Jadida. 
7. MEN (2005b). Wahate riyadiyat, Tronc commun scientifique et technologique. Almadariss.

8. MEN (2006b). Mofid fi riyadiyat (Algèbre et géométrie), lère Année du Baccalauréat sciences mathématiques. Dar Attaqafa.

9. MEN (2008a). Wadih fi riyadiyat (Algèbre et géométrie), lère Année du Baccalauréat sciences mathématiques. Dar Rachad Al Haditha.

10. MEN (2007a). Mofid fi riyadiyat (Analyse), lère Année du Baccalauréat sciences mathématiques. Najah Al Jadida.

11. MEN (2009). Mofid fi riyadiyat (Algèbre et géométrie), 2ème Année du Baccalauréat sciences mathématiques (A et B). Dar Attaqafa.

12. MEN (2008b). Wadih fi riyadiyat (Algèbre et géométrie), 2ème Année du Baccalauréat sciences mathématiques (A et B). Dar Rachad Al Haditha.

13. MEN (2007b). Mofid fi riyadiyat (Analyse), 2ème Année du Baccalauréat sciences mathématiques (A et B). Dar Attaqafa.

14. MEN (2008c). Mostaqbal fi riyadiyat (Analyse), 2ème Année du Baccalauréat sciences mathématiques (A et B). Arrissala.

15. Robert, A. (1999). Recherches didactiques sur la formation professionnelle des enseignants de mathématiques du second degré et leurs pratiques en classe. Didaskalia, 15, 123-157. 